\title{
Analysis of Convergence of the $T$-Transformation for Power Series
}

\section{By Avram Sidi}

\begin{abstract}
Recently the present author has given some convergence theorems of general nature for Levin's nonlinear sequence transformations. In this work these theorems are extended and sharpened to cover the case of power series, both inside and on their circle of convergence. It is shown that one of the two limiting processes considered in the previous work can be used for analytic continuation and a realistic estimate of its rate of convergence is given. Three illustrative examples are also appended.
\end{abstract}

1. Introduction and Review of Recent Results. In a recent paper, Sidi (1979), (from here on denoted as (*)) a partial study of the convergence properties of the nonlinear sequence transformations due to Levin (1973), namely the $T$-transformations, has been given. The purpose of the present work is to extend the results given in (*) to cover the case of power series (and Fourier series), and also to improve upon them. Since we shall be using the notation of $(*)$ and its results, we shall give here its notation and, when needed, those results that are relevant to the present work.

Let the sequence $A_{r}, r=1,2, \ldots$, be a convergent infinite sequence whose limit we denote by $A . T_{k, n}$, the approximation to $A$, and the constants $\gamma_{i}, i=0,1$, $\ldots, k-1$, are defined as the solution of the $k+1$ linear equations

$$
A_{r}=T_{k, n}+R_{r} \cdot \sum_{i=0}^{k-1} \gamma_{i} / r^{i}, \quad r=n, n+1, \ldots, n+k,
$$

where $R_{r}$ are preassigned numbers related to the sequence in consideration; see Levin (1973). Equations (1.1) have a simple solution for $T_{k, n}$ which is given by, see Levin (1973),

$$
T_{k, n}=\frac{\sum_{j=0}^{k}(-1)^{j}\left(\begin{array}{l}
k \\
j
\end{array}\right)(n+j)^{k-1} A_{n+j} / R_{n+j}}{\sum_{j=0}^{k}(-1)^{j}\left(\begin{array}{l}
k \\
j
\end{array}\right)(n+j)^{k-1} / R_{n+j}}
$$

(1.2) can also be written in a more compact and revealing form as, see (*),

$$
T_{k, n}=\frac{\Delta^{k}\left(n^{k-1} A_{n} / R_{n}\right)}{\Delta^{k}\left(n^{k-1} / R_{n}\right)}
$$

where $\Delta$ is the forward difference operator operating on $n$. Once $T_{k, n}$ has been

Received November 28, 1978; revised September 17, 1979.

1980 Mathematics Subject Classification. Primary 41A60, 40A05, 40A25, 41 A25. 
computed, the $\gamma_{i}$ can be computed recursively from, see Theorem 5.1 in (*), (1.4) $\sum_{j=0}^{i} \gamma_{j} \Delta^{k}\left(n^{k+i-j}\right)=\Delta^{k}\left[n^{k+i}\left(A_{n}-T_{k, n}\right) / R_{n}\right], \quad i=0,1, \ldots, k-1$, in this order. Note that $\Delta^{k}$ on the right-hand side of (1.4) does not operate on the index $n$ of $T_{k, n}$.

We now define two limiting processes for $T_{k, n}$;

(1) $k$ is held fixed, $n \rightarrow \infty$ (Process I),

(2) $n$ is held fixed, $k \rightarrow \infty$ (Process II).

In the analysis given in (*) it is assumed that the members of the sequence $\left\{A_{r}\right\}$ satisfy

$$
A_{r}=A+R_{r} f(r), \quad r=1,2, \ldots,
$$

where $f(x)$, as a function of the continuous variable $x$, is defined for all $x \geqslant 1$, including $x=\infty$, and as $x \rightarrow \infty$, has a Poincaré-type asymptotic expansion in inverse powers of $x$, given by

$$
f(x) \sim \sum_{i=0}^{\infty} \beta_{i} / x^{i}, \quad \beta_{0} \neq 0 .
$$

(For Process II it is also assumed that $f(x)$ is an infinitely differentiable function of $x$ for all $x \geqslant 1$ including $x=\infty$.)

Remark 1. If the sequence $A_{r}$ has the above property, then for Process II, which is the more effective of the two processes, $T_{k, n}$ converges to $A$ extremely quickly, as various computations in the literature show. If, on the other hand, the sequence does not possess the above property, then no meaningful results can be expected from $T$, as computations have shown. Therefore, the property above seems to be necessary for $T$ to work at all.

Remark 2. As can be seen easily, if (1.5) and (1.6) are satisfied, then we can express (1.5) in the form

$$
A_{r}=A+\widetilde{R}_{r} \tilde{f}(r), \quad r=1,2, \ldots,
$$

where $\tilde{R}_{r}=R_{r} g(r)$, and $g(x)$, as a function of the continuous variable $x$, as $x \rightarrow \infty$, has a Poincaré-type asymptotic expansion in inverse powers of $x$ like that of $f(x)$ with $\lim _{x \rightarrow \infty} g(x) \neq 0$. Therefore, $\tilde{f}(x)=f(x) / g(x)$ has the same properties as $f(x)$. Thus, by Remark 1 , the $R_{r}$ in (1.2) can be replaced by $\widetilde{R}_{r}$ without affecting $T_{k, n}$ numerically very much.

The observations in Remarks 1 and 2 have been very useful in the derivation of some new numerical quadrature formulas for integrals with algebraic and logarithmic endpoint singularities, which have strong convergence properties. For details see Sidi (1980).

The plan of this paper is as follows: In Section 2 it is shown that for some power series with finite radius of convergence (1.5) and (1.6) hold. Furthermore, the results of (*) are extended to cover the case of some divergent sequences. In Section 3 Process $I$ is analyzed for the power series considered in Section 2 and convergence 
theorems for it are proved. In Section 4 a new approach to Process II is presented, which makes the analysis of this process more amenable. Using this approach, we prove some useful convergence theorems that show, to some extent, the mechanism by which Process II works in some cases including that of power series considered in Section 2, both inside and outside their circle of convergence. The results of Sections 2, 3 and 4 are illustrated with three interesting examples in Section 5.

2. Asymptotic Expansions for Remainders of Some Power Series and Extension of Some Previous Results. Our purpose here is to show, with the help of Theorem 6.1 in (*), under what conditions Levin's transformations can be applied to power series. We begin by recalling Theorem 6.1 of (*), which is a special case of a more general theorem given by Levin and Sidi (1975), for future reference.

THEOREM 2.1 (SEe TheOREM 6.1 of (*)). Let the sequence $A_{r}=\Sigma_{m=1}^{r} a_{m}$, $r=1,2, \ldots$, be such that the terms $a_{r}$ satisfy a linear first-order homogeneous difference equation of the form

$$
a_{r}=p(r) \Delta a_{r}, \quad r=1,2, \ldots,
$$

where $p(x)$, considered as a function of the continuous variable $x$, as $x \rightarrow \infty$, has $a$ Poincaré-type asymptotic expansion in inverse powers of $x$, of the form

$$
p(x) \sim x^{\tau}\left(p_{0}+p_{1} / x+p_{2} / x^{2}+\cdots\right), \quad p_{0} \neq 0,
$$

for $\tau$ an integer $\leqslant 1$. Let $\lim _{r \rightarrow \infty} A_{r}=A, A$ finite. Assume

$$
\lim _{r \rightarrow \infty} p(r) a_{r}=0
$$

and

$$
\overline{l p} \neq 1, \quad l=-1,1,2,3, \ldots,
$$

where $\bar{p}=\lim _{x \rightarrow \infty} p(x) / x$. Then $A-A_{r_{-1}}$, as $r \rightarrow \infty$, has an asymptotic expansion of the form

$$
A-A_{r-1}=\sum_{m=r}^{\infty} a_{m} \sim a_{r} r^{\tau}\left(\beta_{0}^{\prime}+\beta_{1}^{\prime} / r+\beta_{2}^{\prime} / r^{2}+\cdots\right) .
$$

Furthermore, from the constructive proof of this theorem it follows that $\beta_{0}^{\prime}=$ $-p_{0} /(p+1) \neq 0$.

If we now subtract $a_{r}$ from both sides of (2.5) and rearrange, we obtain

$$
A_{r} \sim A+a_{r} r^{\tau}\left(\beta_{0}+\beta_{1} / r+\beta_{2} / r^{2}+\cdots\right) \text {, }
$$

where

$$
\beta_{i}=\left\{\begin{array}{ll}
-\beta_{i}^{\prime} & \text { if } i \neq \tau, \\
-\beta_{i}^{\prime}+1 & \text { if } i=\tau,
\end{array} \quad i=0,1,2, \ldots .\right.
$$

Remark 1. It follows from (2.6), (1.5) and (1.6) that a very natural way to choose $R_{r}$ is by letting $R_{r}=a_{r} r^{\tau}$; see Levin (1973). 
It turns out that there is a large class of infinite power series satisfying the conditions of Theorem 2.1, as the following theorem shows.

THEOREM 2.2. Let $A_{r}=\Sigma_{m=1}^{r} a_{m}, r=1,2, \ldots$, and suppose $a_{r}$ is of the form

$$
a_{r}=z^{r-1} w(r)
$$

where $z$ is a complex parameter, $z \in D \subset C$, and $w(x)$, as a function of the continuous variable $x$, as $x \rightarrow \infty$, has a Poincaré-type asymptotic expansion of the form

$$
w(x) \sim x^{\alpha}\left(w_{0}+w_{1} / x+w_{2} / x^{2}+\cdots\right), \quad w_{0} \neq 0 .
$$

Then all the conditions of Theorem 2.1 are satisfied simultaneously for (1) $D=\{z|| z \mid$ $<1\}$ with any $\alpha$, (2) $D=\{z|| z \mid \leqslant 1, z \neq 1\}$ with $\alpha<0$, and (3) $D=\{z|| z \mid \leqslant 1\}$ with $\alpha<-1$. Hence an asymptotic expansion of the form (2.6) exists.

Remark 2. The infinite series $\Sigma_{r=1}^{\infty} a_{r}=\Sigma_{r=1}^{\infty} w(r) z^{r-1}$ represents an analytic function $F(z)$ in the open disc $|z|<1$. The point $z=1$ is usually a point of singularity of this function, a branch point or a pole, while other points on the unit circle are regular points.

Proof. As can be easily seen from (2.8) and (2.9), the radius of convergence of the infinite series $\Sigma_{r=1}^{\infty} a_{r}$ is 1 for all values of $\alpha$, which explains (1). The convergence of the infinite series on the unit circle with the exception of $z=1$ explains (2). The convergence of the infinite series everywhere on the unit circle explains (3). Hence (1), (2), (3) guarantee the convergence of $A_{r}$ for the specified ranges of $z$.

Now the terms $a_{r}$ satisfy a difference equation of the form (2.1), where $p(r)$ is simply

$$
p(r)=\left(a_{r+1} / a_{r}-1\right)^{-1},
$$

therefore, $1 / p(x)=z w(x+1) / w(x)-1$. Now $1 / p(x)$, as $x \rightarrow \infty$, has a Poincarétype asymptotic expansion which can be shown to be

$$
1 / p(x)=(z-1)+\alpha z / x+O\left(x^{-2}\right) .
$$

Hence $p(x)$, as $x \rightarrow \infty$, has a Poincaré-type asymptotic expansion which is given by

$$
p(x)= \begin{cases}1 /(z-1)-\left[\alpha z /(z-1)^{2}\right] / x+O\left(x^{-2}\right), & z \neq 1, \\ x / \alpha+O(1), & z=1 .\end{cases}
$$

From (2.12) it follows that $\tau=0$, hence $\bar{p}=0$, whenever $z \neq 1$ and $\tau=1$ with $\bar{p}=$ $1 / \alpha$ for $z=1$, where $\bar{p}$ and $\tau$ have been defined in Theorem 2.1. Using these last results it is easy to verify that (2.3) and (2.4) are satisfied. This completes the proof of the theorem.

If we now choose $R_{r}$, as explained in the remark following Theorem 2.1 , then we have

$$
R_{r}=z^{r-1} g(r)
$$


where $g(r)=w(r) r^{\tau}$. Considered as a function of the continuous variable $x$, as $x \rightarrow \infty, g(x)$ has a Poincare-type asymptotic expansion of the form

$$
g(x) \sim x^{\alpha+\tau}\left(w_{0}+w_{1} / x+w_{2} / x^{2}+\cdots\right) .
$$

We note that for this case $R_{r}$ can also be taken as

$$
R_{r}=z^{r-1} r^{\alpha+\tau},
$$

which is the dominant term of $A_{r}-F(z)$ as $r$ becomes large, in accordance with Remark 2 in Section 1. In the derivation of the new numerical quadrature formulas in Sidi (1980), the $R_{r}$ used are of the form given in (2.15) and not (2.13).

Remark 3. When the $T$-transformation is applied to a power series, $T_{k, n}$ turns out to be a rational function

In many cases the relation

$$
A_{r}=F(z)+R_{r} f(r)
$$

together with (1.6), whose existence, whenever $\lim _{r \rightarrow \infty} A_{r}$ exists, has been proved above, can be continued analytically to the unit circle and its exterior. (Examples of this will be given later.) That is, in some cases (2.16) is valid even when $\lim _{r \rightarrow \infty} A_{r}$ does not exist. (We recall that in Theorem 2.1 we assumed the existence of $\lim _{r \rightarrow \infty} A_{r}$ )

In view of the remark above, we now extend Theorems 3.1 and 3.2 in (*) to cover also the case of some nonconvergent sequences as follows:

THEOREM 2.3. Let the sequence $A_{r} r=1,2, \ldots$, (convergent or not) satisfy (1.5), where $f(x)$ is as explained in Section 1 and satisfies (1.6). If, in addition, $R_{n}=$ $O\left(n^{\alpha}\right)$ for some $\alpha$ as $n \rightarrow \infty$, and, for $k>\alpha, k$ fixed,

$$
\sup _{n}\left|\Delta^{k}\left((-1)^{n} n^{k-1} /\left|R_{n}\right|\right) / \Delta^{k}\left(n^{k-1} / R_{n}\right)\right|<\infty,
$$

then $T_{k, n} \rightarrow A$ as $n \rightarrow \infty ;$ actually $T_{k, n}-A=O\left(n^{-k+\alpha}\right)$.

This theorem extends Corollary 2 of Theorem 3.1 in (*), and its proof is similar to that given in (*).

THEOREM 2.4. Let the sequence $A_{r} r=1,2, \ldots$, (convergent or not), $f(x)$, and $R_{n}$ be as in Theorem 2.3 and assume that $f(x)$ is infinitely differentiable for all $x \geqslant n$, including $x=\infty$. If, for $n$ fixed,

$$
\sup _{k}\left|\Delta^{k}\left((-1)^{n} n^{k-1} /\left|R_{n}\right|\right) / \Delta^{k}\left(n^{k-1} / R_{n}\right)\right|<\infty
$$

then $T_{k, n} \rightarrow A$ as $k \rightarrow \infty$; actually $T_{k, n}-A=o\left(k^{-\lambda}\right)$ for any $\lambda \geqslant 0$.

This theorem extends Corollary 2 of Theorem 3.2 in (*), and its proof is similar to that given in $(*)$.

As in (*), these last theorems can be applied immediately to oscillatory sequences for which $R_{r} R_{r+1}<0, r=1,2, \ldots$, since for these sequences 


$$
\left|\Delta^{k}\left((-1)^{n} n^{k-1} /\left|R_{n}\right|\right) / \Delta^{k}\left(n^{k-1} / R_{n}\right)\right|=1,
$$

see (*), giving us an extension of Theorem 4.1 in (*).

THEOREM 2.5. Let the sequence $A_{r}, r=1,2, \ldots$, (convergent or not), $f(x)$, and $R_{n}$ be as described in Theorem 2.3 and assume $R_{r} R_{r+1}<0, r=1,2, \ldots$. Then when $k>\alpha$, $k$ fixed, $T_{k, n}-A=O\left(n^{-k+\alpha}\right)$ as $n \rightarrow \infty$. If in addition $f(x)$ is infinitely differentiable as described in Theorem 2.4, then, for $n$ fixed, $T_{k, n}-A=$ $o\left(k^{-\lambda}\right)$ as $k \rightarrow \infty$ for any $\lambda \geqslant 0$.

The above theorems can now be applied to the power series that have been considered in Theorem 2.2 and the remark following it, inside and on the circle of convengence. Especially when $z=-1$, Theorem 2.5 can be applied to the partial sums of the infinite series $\Sigma_{m=1}^{\infty}(-1)^{m-1} w(m)$, where $w(m)>0$ for all $m$ and $w(x)$ is as in (2.9).

Although the results of Theorems 2.3-2.5 are stronger than their predecessors given in (*), they are still not the best, due to their general nature. In the next sections, we shall improve on them by making certain (realistic) assumptions about the sequences to which Levin's transformations are applied.

3. Application of Process I to Power Series and Fourier Series. The purpose of this section is to extend Theorems 4.2 and 5.2 of (*), which were stated and proved for some monotone sequences, to cover the case of infinite power series such as those that we have considered in the previous section, inside and on the unit circle, taking into account Remark 2 in Section 2. Our new results will be stated in slightly more general terms. They seem to be the best that one can obtain under the given conditions.

THEOREM 3.1. Let the sequence $A_{r} r=1,2, \ldots$, (convergent or divergent) depending on the complex parameter $z$, satisfy

$$
A_{r}=F(z)+R_{r} f(r),
$$

where $F(z)$ is a function depending on $z$ such that $\lim _{r \rightarrow \infty} A_{r}=F(z)$ whenever this limit exists, and

$$
R_{r}=z^{r-1} g(r)
$$

where $g(x)$, as a function of the continuous variable $x$, when $z \neq 1$ has a Poincarétype asymptotic expansion of the form

$$
g(x) \sim \sum_{i=0}^{\infty} \rho_{i} / x^{i+\sigma}, \quad \rho_{0} \neq 0,
$$

and $f(x)$, considered as a function of the continuous variable $x$, has a Poincaré-type asymptotic expansion of the form (1.6) with the same notation. Let $T_{k, n}$ be as given in (1.3). Then, when $z \neq 1$,

$$
T_{k, n}-F(z)=z^{n-1} / n^{2 k+\sigma}\left[D+O\left(n^{-1}\right)\right] \text { as } n \rightarrow \infty
$$


where

$$
D=\rho_{0} \beta_{k} k !(1-1 / z)^{-k} .
$$

Proof. Equation (3.6) in (*) reads

$$
T_{k, n}-F(z)=\frac{\Delta^{k}\left[n^{k-1} w_{k}(n)\right]}{\Delta^{k}\left(n^{k-1} / R_{n}\right)}
$$

where

$$
w_{k}(x)=f(x)-\sum_{i=0}^{k-1} \beta_{i} / x^{i}
$$

Now $w_{k}(x)=\beta_{k} / x^{k}+O\left(x^{-k-1}\right)$ as $x \rightarrow \infty$, therefore $x^{k-1} w_{k}(x)=\beta_{k} / x+O\left(x^{-2}\right)$ as $x \rightarrow \infty$, consequently $\Delta^{k}\left[n^{k-1} w_{k}(n)\right]=O\left(n^{-k-1}\right)$ as $n \rightarrow \infty$. Using the fact that

$$
\Delta^{k} n^{-1}=(-1)^{k} k ! /[n(n+1) \cdots(n+k)],
$$

which can easily be proved by induction, we can actually write for the numerator of

$$
\Delta^{k}\left[n^{k-1} w_{k}(n)\right]=(-1)^{k} k ! / n^{k+1}\left[\beta_{k}+O\left(n^{-1}\right)\right] \quad \text { as } n \rightarrow \infty .
$$

As for the denominator of (3.6) we proceed as follows: since $g(x)$ has a Poincarétype asymptotic expansion, so does $1 / g(x)$ and its asymptotic expansion is given by

$$
1 / g(x) \sim \sum_{i=0}^{\infty} \epsilon_{i} x^{\sigma-i} \text { as } x \rightarrow \infty,
$$

where $\epsilon_{0}=1 / \rho_{0}$. We now need the asymptotic behavior of $\Delta^{k}\left(z^{-n} n^{\alpha}\right)$ as $n \rightarrow \infty$. First of all we have

$$
\Delta^{k}\left(z^{-n} n^{\alpha}\right)=\sum_{j=0}^{k}(-1)^{k-j}\left(\begin{array}{l}
k \\
j
\end{array}\right)(n+j)^{\alpha} z^{-n-j},
$$

which, as $n \rightarrow \infty$, can be shown to behave like

$$
\begin{aligned}
\Delta^{k}\left(z^{-n} n^{\alpha}\right) & =\left[\sum_{j=0}^{k}(-1)^{k-j}\left(\begin{array}{l}
k \\
j
\end{array}\right) z^{-j}\right] z^{-n} n^{\alpha}\left[1+O\left(n^{-1}\right)\right] \\
& =(-1)^{k}(1-1 / z)^{k} z^{-n} n^{\alpha}\left[1+O\left(n^{-1}\right)\right] .
\end{aligned}
$$

Combining (3.10) and (3.12), we obtain for the denominator of (3.6)

$$
\begin{aligned}
\Delta^{k}\left(n^{k-1} / R_{n}\right) & =\Delta^{k}\left[n^{k-1} z^{-n+1} \epsilon_{0} n^{\sigma}\left(1+O\left(n^{-1}\right)\right)\right] \\
& =(-1)^{k}(1-1 / z)^{k} \epsilon_{0} z^{-n+1} n^{k+\sigma-1}\left[1+O\left(n^{-1}\right)\right] \quad \text { as } n \rightarrow \infty .
\end{aligned}
$$

Substituting now (3.9) and (3.13) in (3.6) and using the fact that $\epsilon_{0}=1 / \rho_{0}$, we obtain (3.4) together with (3.5), thus proving the theorem.

Corollary. If $|z| \leqslant 1, z \neq 1$, then $T_{k, n} \rightarrow F(z)$, as $n \rightarrow \infty$, provided $k$ is chosen so that $2 k+\sigma>0$. For $|z|>1$, however, $T_{k, n}$ diverges as $n \rightarrow \infty$, i.e., Process I cannot be used for analytic continuation beyond the circle of convergence of the infinite series considered in Section 2. 
Proof. The proof follows by observing that the right-hand side of (3.4) tends to zero as $n \rightarrow \infty$ for $|z| \leqslant 1, z \neq 1$, only if $2 k+\sigma>0$. For $|z|>1$, however $T_{k, n}-F(z)=O\left(z^{n}\right)$ as $n \rightarrow \infty$, thus completing the proof.

Remark 1. (3.1), (3.2) and (3.3) imply that $\lim _{r \rightarrow \infty} A_{r}=F(z)$ for (1) $|z|<1$ for all $\sigma$, and (2) $|z| \leqslant 1$ for $\sigma>0$. The corollary above tells us that $T_{k, n} \rightarrow F(z)$ as $n \rightarrow \infty$ for all $|z| \leqslant 1, z \neq 1$, no matter what $\sigma$ is, i.e., whether $\lim _{r \rightarrow \infty} A_{r}$ exists or not, provided $k$ is chosen large enough so that $2 k+\sigma>0$.

Remark 2. Equation (3.4) tells us that for $z \neq 1$, whenever $A_{n}$ converges to $F(z)$ as $n \rightarrow \infty, T_{k, n}$ converges to $F(z)$ more quickly, in fact

$$
\frac{T_{k, n}-F(z)}{A_{n}-F(z)}=O\left(n^{-2 k}\right) \quad \text { as } n \rightarrow \infty \text {. }
$$

Remark 3. From the expression for $D$, given in (3.5), we can see that problems will arise as we approach $z=1$. Indeed, there is a drastic fall in the rate of convergence of $T_{k, n}$ to $F(z)$, as numerical experiments show. Also Theorem 4.2 in (*) shows that, if $\lim _{r \rightarrow \infty} A_{r}=F(1)$ exists, we have

$$
\frac{T_{k, n}-F(1)}{A_{n}-F(1)}=O\left(n^{-k}\right) \quad \text { as } n \rightarrow \infty,
$$

as opposed to (3.14).

Going back to $A_{r}=\Sigma_{m=1}^{r} w(m) z^{m-1}$, where $w(x)$ is as described in the previous section, we can see that, on $|z|=1, A_{r}$ is a partial sum of the complex Fourier series $\Sigma_{m=1}^{\infty} w(m) e^{i(m-1) \theta}$, where we have put $z=e^{i \theta}$. Hence Theorems 2.2 and 3.1 cover the case of the complex Fourier series, whose coefficients $w(m)$ are as described in Section 2.

THEOREM 3.2. Let the sequence $A_{r} r=1,2, \ldots$, satisfy all the conditions of Theorem 3.1 with the notation therein and let $\gamma_{i}, i=0,1, \ldots, k-1$, be as in (1.1). Then, for $z \neq 1$, we have

$$
\gamma_{i}-\beta_{i}=O\left(n^{-k+i}\right) \text { as } n \rightarrow \infty .
$$

Proof. The proof of (3.16) proceeds along the same lines as that of Theorem 5.2 in (*). Equation (5.6) in (*) reads

$$
\begin{aligned}
{\left[F(z)-T_{k, n}\right] \Delta^{k}\left(n^{k+i} / R_{n}\right)+\Delta^{k}\left[n^{k+i} f(n)\right] } & \\
& =\sum_{j=0}^{i} \gamma_{j} \Delta^{k}\left(n^{k+i-j}\right), \quad i=0,1, \ldots, k-1 .
\end{aligned}
$$

Now $\Delta^{k}\left(n^{k+i} / R_{n}\right)=z^{-n+1} O\left(n^{k+i+\sigma}\right)$ as $n \rightarrow \infty$, which can be proved in a way similar to that in Theorem 3.1. Also $F(z)-T_{k, n}=z^{n-1} O\left(n^{-2 k-\sigma}\right)$ as $n \rightarrow \infty$ which follows from (3.4). Therefore, the first term on the left-hand side of (3.17) is just

$$
\left[F(z)-T_{k, n}\right] \Delta^{k}\left(n^{k+i} / R_{n}\right)=O\left(n^{-k+i}\right) \text { as } n \rightarrow \infty .
$$

Once this has been established the rest of the proof is exactly the same as that of Theorem 5.2 in (*), therefore we shall omit it. 
We note that Theorem 5.2 in (*) covers the case $z=1$ and for this case too $\gamma_{i}-\beta_{i}=O\left(n^{-k+i}\right), i=0, \ldots, k-1$.

4. Another Approach to the Analysis of Levin's Transformations. In Theorem 2.4 it was assumed that the sequence $A_{r} r=1,2, \ldots$, (convergent or not) satisfies (1.5), where $f(x)$, as a function of the continuous variable $x$, is defined and is infinitely differentiable for all $x \geqslant 1$, including $x=\infty$, and has a Poincaré-type asymptotic expansion of the form (1.6). We shall now assume further that $f(x) / x=\bar{f}(x)$ is the Laplace transform of a function $\phi(t)$, which is an infinitely differentiable function of $t$ for $0 \leqslant t<\infty$, i.e.,

$$
\bar{f}(x)=L[\phi(t) ; x]=\int_{0}^{\infty} e^{-x t} \phi(t) d t .
$$

Then, using Watson's lemma, see Olver (1974, p. 71), we have

$$
\bar{f}(x) \sim \sum_{i=0}^{\infty} \phi^{(i)}(0) / x^{i+1} \quad \text { as } x \rightarrow \infty,
$$

where we immediately identify $\phi^{(i)}(0)$ as $\beta_{i}$. (Examples of this will be given in Section 5.)

Equation (3.7) in (*) reads

$$
T_{k, n}-A=\frac{\Delta^{k}\left[n^{k-1} f(n)\right]}{\Delta^{k}\left(n^{k-1} / R_{n}\right)}
$$

which, in view of the assumptions above, can be expressed as

$$
T_{k, n}-A=\frac{\Delta^{k}\left[n^{k} \bar{f}(n)\right]}{\Delta^{k}\left(n^{k-1} / R_{n}\right)}
$$

Now, from the theory of the Laplace transform we have, see Sneddon (1972, p. 147),

$$
L\left[\phi^{(m)}(t) ; x\right]=x^{m} \bar{f}(x)-\sum_{i=0}^{m-1} \phi^{(m-i-1)}(0) x^{i} .
$$

Letting $x=n, m=k$, and applying the operator $\Delta^{k}$ to both sides of (4.5) and using the fact that $\Delta^{k} p(n)=0$, when $p(n)$ is a polynomial in $n$ of degree at most $k-1$, we obtain

$$
\Delta^{k}\left[n^{k} \bar{f}(n)\right]=\Delta^{k}\left\{L\left[\phi^{(k)}(t) ; n\right]\right\}=\Delta^{k}\left[\int_{0}^{\infty} e^{-n t} \phi^{(k)}(t) d t\right]
$$

Since the operator $\Delta^{k}$ operates only on $n$ and since

$$
\Delta^{k}\left(e^{-n t}\right)=e^{-n t}\left(e^{-t}-1\right)^{k},
$$

we can express $(4.6)$ in the form

$$
\Delta^{k}\left[n^{k} \bar{f}(n)\right]=\int_{0}^{\infty} e^{-n t}\left(e^{-t}-1\right)^{k} \phi^{(k)}(t) d t .
$$

We have therefore proved the following 
THEOREM 4.1. Let the sequence $A_{r} r=1,2, \ldots$, (convergent or not) be as described in the first paragraph of this section. Then

$$
T_{k, n}-A=\frac{L\left[\left(e^{-t}-1\right)^{k} \phi^{(k)}(t) ; n\right]}{\Delta^{k}\left(n^{k-1} / R_{n}\right)} .
$$

If Eq. (4.9) is used in the analysis of Process I ( $k$ fixed, $n \rightarrow \infty$ ), it seems that one can obtain only those results that were given previously, so that there is not much to be gained from (4.9), as far as Process I is concerned.

As for Process II ( $n$ fixed, $k \rightarrow \infty$ ), which is the more effective of the two processes, yet the more difficult to analyze, Theorem (4.1) does seem to represent a breakthrough. Of course, eventually one has to analyze the asymptotic behavior of $L\left[\left(e^{-t}-1\right)^{k} \phi^{(k)}(t) ; n\right]$ and of $\Delta^{k}\left(n^{k-1} / R_{n}\right)$ as $k \rightarrow \infty$, which is not an easy task in general. The following results and the examples in the next section do, however, give an indication about the mechanism by which Process II works and why it works so efficiently.

LEMмA 4.1. Let $\phi(\xi)$ be analytic and uniformly bounded in the half strip $S(u)$ $=\{\xi|\operatorname{Re} \xi \geqslant-u,| \operatorname{Im} \xi \mid \leqslant u\}$, for some $u>0$. Then

$$
\left|L\left[\left(e^{-t}-1\right)^{k} \phi^{(m)}(t) ; n\right]\right| \leqslant M m ! k ! /\left[u^{m+1} n(n+1) \cdots(n+k)\right],
$$

where $M$ is the uniform bound of $\phi(\xi)$ in $S(u)$; i.e., $|\phi(\xi)| \leqslant M$ for $\xi \in S(u)$.

Proof. Since $\phi(\xi)$ is analytic in $S(u)$, we can write, using Cauchy's formulas,

$$
\phi^{(m)}(t)=\frac{m !}{2 \pi i} \int_{|\xi-t|=u} \frac{\phi(\xi)}{(\xi-t)^{m+1}} d \xi,
$$

where $t \in[0, \infty)$. Taking the modulus of both sides of (4.11) and using the assumption of uniform boundedness, we obtain

$$
\left|\phi^{(m)}(t)\right| \leqslant M m ! / u^{m+1} .
$$

Making use of (4.12), we therefore have

$$
\left|L\left[\left(e^{-t}-1\right)^{k} \phi^{(m)}(t) ; n\right]\right| \leqslant M m ! / u^{m+1} \int_{0}^{\infty} e^{-n t}\left(1-e^{-t}\right)^{k} d t .
$$

But

$$
\begin{aligned}
\int_{0}^{\infty} e^{-n t}\left(1-e^{-t}\right)^{k} d t & =(-1)^{k} \Delta^{k}\left(\int_{0}^{\infty} e^{-n t} d t\right) \\
& =(-1)^{k} \Delta^{k}\left(n^{-1}\right)=k ! /[n(n+1) \cdots(n+k)]
\end{aligned}
$$

by (3.8). Substituting (4.14) in (4.13), (4.10) now follows.

COROllary. If $m=k+p$, where $p$ is fixed, then

$$
\left|L\left[\left(e^{-t}-1\right)^{k} \phi^{(k+p)}(t) ; n\right]\right| \leqslant \bar{M} k ! /\left(u^{k} k^{n-p}\right),
$$

for some constant $\bar{M}>0$ which is independent of $k$.

The proof of (4.15) follows easily from (4.10). 
We shall now apply Theorem 4.1 and the corollary of Lemma 4.1 to the power series considered in Sections 2 and 3.

THEOREM 4.2. Let the sequence $A_{r}, r=1,2, \ldots$, be as in Theorems 3.1 and 4.1 and Lemma 4.1 with the notation therein. Then, for $z$ real and negative and $|z|<$ $(u e)^{2}$,

$$
T_{k, n}-F(z)=O\left(q^{-k} k^{-n-\sigma+2}\right) \quad \text { as } k \rightarrow \infty
$$

at least, where $q=u e|z|^{-1 / 2}>1$.

Proof. From the conditions above it is clear that (4.15) holds, therefore the numerator of the expression on the right-hand side of (4.9) is at least $O\left(k ! k^{-n} u^{-k}\right)$ as $k \rightarrow \infty$. As for the denominator of this expression we proceed as follows: Since $g(m)$ satisfies (3.3), $g(m) \sim \rho_{0} m^{-\sigma}$ as $m \rightarrow \infty$ and has a fixed sign (that of $\rho_{0}$ ) for $m \geqslant m_{0}$ for some positive integer $m_{0}$. Denoting

$$
\begin{aligned}
b_{j} & =(-1)^{j}\left(\begin{array}{l}
k \\
j
\end{array}\right)(n+j)^{k-1} / R_{n+j} \\
& =(-1)^{n+1}\left(\begin{array}{l}
k \\
j
\end{array}\right)(n+j)^{k-1}|z|^{-n-j+1} / g(n+j), \quad j=0,1,2, \ldots, k,
\end{aligned}
$$

we can write for the denominator

$$
\left|\sum_{j=0}^{k} b_{j}\right| \geqslant\left\|\sum_{j=0}^{m_{0}-1} b_{j}|-| \sum_{j=m_{0}}^{k} b_{j}\right\| .
$$

Now since $b_{j}$ are all of the same sign for $j \geqslant m_{0}$, and $m_{0}$ is fixed, we can write

$$
\begin{aligned}
\left|\sum_{j=m_{0}}^{k} b_{j}\right|>\left|b_{[k / 2]}\right| & =\left(\begin{array}{c}
k \\
{[k / 2]}
\end{array}\right)\left(n+\left[\frac{k}{2}\right]\right)^{k-1}|z|^{-n-[k / 2]+1} / g\left(n+\left[\frac{k}{2}\right]\right) \\
& =O\left[k !\left(\left.\left.e\right|_{z}\right|^{-1 / 2}\right)^{k} k^{\sigma-2}\right] \text { as } k \rightarrow \infty,
\end{aligned}
$$

which can be proved by using Stirling's formula, $k ! \sim k^{k} e^{-k} \sqrt{2 \pi k}$ as $k \rightarrow \infty$. Essentially, this is a $O\left(k^{k}\right)$-like behavior. The sum $\left|\sum_{j=0}^{m} 0^{-1} b_{j}\right|$, on the other hand, can grow at most like $k^{m_{0}}\left(n+m_{0}\right)^{k}$ as $k \rightarrow \infty$ as can readily be verified. Therefore,

$$
\left|\sum_{j=0}^{k} b_{j}\right| \sim\left|\sum_{j=m_{0}}^{k} b_{j}\right| \text { as } k \rightarrow \infty .
$$

Combining these results for the numerator and denominator in (4.9), the result follows.

Remark 1. By replacing (4.19) by

$$
\left|\sum_{j=m_{0}}^{k} b_{j}\right|>\left|b_{[\alpha k]}\right|, \quad \frac{m_{0}}{k}<\alpha<1,
$$

we can show, by using the method above, that (4.16) holds with $q=$ $[\alpha /(1-\alpha)]^{1-\alpha} u e|z|^{-\alpha}$, provided $z$ is chosen such that $q>1$. Now one can choose $\alpha$ so as to make $q$ as large as possible.

We now give a result that will be useful in dealing with monotonic sequences. 
THEOREM 4.3. Let the sequence $A_{r} r=1,2, \ldots$, be as in Theorem 4.1 and Lemma 4.1 with $u>1$ and the notation therein, and with $R_{r}=r^{-\alpha}$, for some $\alpha>0$. Then

$$
T_{k, n}-A=O\left(u^{-k} k^{-n-\alpha+2}\right) \text { as } k \rightarrow \infty
$$

Remark 2. Such a sequence is monotonic. If $A_{r}$ are the partial sums of the power series considered in Theorem 2.2, then $R_{r}=r^{-\alpha}$ corresponds to the case $z=1$ and for this case a finite limit exists, if $\alpha>0$. Otherwise the limit is infinite.

Proof. As in Theorem 4.2, the numerator of the expression on the right-hand side of (4.9) is at least $O\left(k ! k^{-n} u^{-k}\right)$ as $k \rightarrow \infty$. Now the denominator of this expression becomes $\Delta^{k}\left(n^{k+\alpha-1}\right)$. From the calculus of finite differences we know that, see Isaacson and Keller (1966, p. 262),

$$
\Delta^{k} h(x)=h^{(k)}(y) \text { for some } y \in(x, x+k)
$$

Therefore,

$$
\Delta^{k} x^{\sigma}=\left[\prod_{j=0}^{k-1}(\sigma-j)\right] y^{\sigma-k} \quad \text { for some } y \in(x, x+k) .
$$

Hence the denominator becomes

$$
\Delta^{k}\left(n^{k+\alpha-1}\right)=\frac{(k+\alpha-1) !}{(\alpha-1) !} m^{\alpha-1} \quad \text { for some } m \in(n, n+k) .
$$

Using Stirling's approximation, we have $(k+\alpha-1) ! \sim B k ! k^{\alpha-1}$ as $k \rightarrow \infty$, for some $B>0$ independent of $k$. Also $m^{\alpha-1}>n^{\alpha} /(n+k)$. Combining all these results in (4.9), (4.21) follows.

We now consider the $\gamma_{i}$ in (1.1).

THEOREM 4.4. If the sequence $A_{r} r=1,2, \ldots$, is as in Theorem 4.1 , then

$$
\begin{aligned}
\sum_{j=0}^{i}\left(\gamma_{j}-\beta_{j}\right) \Delta^{k}\left(n^{k+i-j}\right)= & {\left[A-T_{k, n}\right] \Delta^{k}\left(n^{k+i} / R_{n}\right) } \\
& +L\left[\left(e^{-t}-1\right)^{k} \phi^{(k+i+1)}(t) ; n\right], \\
& i=0,1, \ldots, k-1 .
\end{aligned}
$$

Proof. Using (3.17), we just have to prove that

$$
\begin{aligned}
\Delta^{k}\left[n^{k+i} f(n)\right]= & L\left[\left(e^{-t}-1\right)^{k} \phi^{(k+i+1)}(t) ; n\right] \\
& +\sum_{j=0}^{i} \beta_{j} \Delta^{k}\left(n^{k+i-j}\right), \quad i=0,1, \ldots, k-1 .
\end{aligned}
$$

This can be proved easily by using (4.5) with $x=n$ and $m=k+i+1$ and applying $\Delta^{k}$ to both sides, keeping in mind that $\Delta^{k} p(n)=0$ when $p(n)$ is a polynomial of degree at most $k-1$ and that $\beta_{j}=\phi^{(j)}(0), j=0,1, \ldots$. 
THEOREM 4.5. Let the sequence $A_{r}, r=1,2, \ldots$, be as in Theorem 4.3 with the notation therein. Then, for fixed $i$,

$$
\gamma_{i}-\beta_{i}=O\left(u^{-k} k^{p_{i}}\right) \text { as } k \rightarrow \infty,
$$

where $p_{i}$ depends on $n, \alpha$, and $i$.

Proof. We shall prove (4.27) by induction on $i$. For $i=0,(4.25)$ becomes

$$
\left(\gamma_{0}-\beta_{0}\right)=\left[A-T_{k, n}\right] \frac{\Delta^{k}\left(n^{k} / R_{n}\right)}{k !}+\frac{L\left[\left(e^{-t}-1\right)^{k} \phi^{(k+1)}(t) ; n\right]}{k !} .
$$

Now $\left[A-T_{k, n}\right]=O\left(u^{-k} k^{-n-\alpha+2}\right)$ as $k \rightarrow \infty$. From (4.23) $\Delta^{k}\left(n^{k} / R_{n}\right)=$ $\Delta^{k}\left(n^{k+\alpha}\right)=m^{\alpha}(k+\alpha) ! / \alpha$ ! for some $m \in(n, n+k)$. Using Stirling's formula, we obtain $\Delta^{k}\left(n^{k} / R_{n}\right)=O\left(k ! k^{2 \alpha}\right)$ as $k \rightarrow \infty$. Therefore, the first term on the righthand side of (4.28) is $O\left(u^{-k} k^{-n+\alpha+2}\right)$ as $k \rightarrow \infty$. Using (4.15) in the corollary of Lemma 4.1, we can see that the second term is $O\left(u^{-k} k^{-n+1}\right)$ as $k \rightarrow \infty$. Hence we have shown that (4.27) holds for $i=0$, with $p_{0}=-n+\alpha+2$. Let us now assume that (4.27) is true for $i \leqslant m-1$. For $i=m$ we have from (4.25),

$$
\begin{aligned}
\gamma_{m}-\beta_{m}= & {\left[A-T_{k, n}\right] \frac{\Delta^{k}\left(n^{k+m} / R_{n}\right)}{k !}+\frac{L\left[\left(e^{-t}-1\right)^{k} \phi^{(k+m+1)}(t) ; n\right]}{k !} } \\
& +\sum_{j=0}^{m-1}\left(\gamma_{j}-\beta_{j}\right) \frac{\Delta^{k}\left(n^{k+i-j}\right)}{k !} .
\end{aligned}
$$

Using in (4.29) the same technique that was used in (4.28), we again have $\gamma_{m}-\beta_{m}=$ $O\left(u^{-k} k^{p_{m}}\right)$ as $k \rightarrow \infty$, where $p_{m}$ depends on $n, \alpha$, and $m$. This proves the theorem.

In many interesting cases it can be shown that $\bar{f}(x)$ is a Laplace transform as in (4.1) and that $\phi(\xi)$ satisfies the conditions of Lemma 4.1 so that (4.10) and hence (4.15) hold. These points will be illustrated with three typical examples in the next section.

Before closing this section, we note that Wimp (1977) has considered the problem of accelerating the convergence of some monotonic sequences of the form similar to that considered in this section, with $R_{r}=r^{-1}$. Wimp develops different transformations, in the form of linear summability methods, corresponding to different $L_{p}^{s}(0, \infty)$ classes of the function $\phi(t)$. (It is assumed that $(1) \phi(t) \in L_{1}(0, \infty)$, (2) $\phi^{(s)}(t)$ is locally integrable on $(0, \infty)$, and (3) $\phi^{(s)}(t) e^{\epsilon c t} \in L_{p}(0, \infty)$ for some $\epsilon>0$, $0<c<1$.) For finite $s$, for which

$$
\bar{f}(x)=\sum_{i=0}^{s-1} \frac{\phi^{(i)}(0)}{x^{i+1}}+o\left(\frac{1}{x^{s}}\right) \text { as } x \rightarrow \infty,
$$

useful error bounds and rates of convergence are provided. For $s=\infty$, which is the case considered also in the present work and in (*), though with stronger assumptions on $\phi(t)$, Wimp's method gives approximations which are very similar to $T_{k, n}$ with $R_{r}=r^{-1}$. Actually Levin's $T$-transformation for the sequence 
$A_{r} r=1,2, \ldots$, with $R_{r}=r^{-1}$ reduces to

$$
T_{k, n}=\sum_{j=0}^{k}(-1)^{k+j}\left(\begin{array}{l}
k \\
j
\end{array}\right) \frac{(n+j)^{k}}{k !} A_{n+j},
$$

whereas Wimp's transformation for the sequence $B_{j}, j=0,1, \ldots$, is

$$
\bar{B}_{k}=\sum_{j=0}^{k}(-1)^{k+j}\left(\begin{array}{l}
k \\
j
\end{array}\right) \frac{(\epsilon+j)^{k}}{k !} B_{j},
$$

where $\bar{B}_{k}, k=0,1, \ldots$, is the sequence of approximations to $B=\lim _{j \rightarrow \infty} B_{j}$. As is clear from above, for Wimp's transformation Process I does not exist. For the case $s=\infty$ Wimp gives bounds for $B-\bar{B}_{k}$ but makes no statement about convergence or rate of convergence as $k \rightarrow \infty$. It seems that no such statement can be made, if no new assumptions are made on $\phi(t)$, except its being an $L_{p}^{\infty}$ function.

With the assumptions that we have made on $\phi(\xi)$ in the complex $\xi$-plane, we have been able to prove results on convergence and rates of convergence for the $T$ transformation with different types of $R_{r}$ and in particular with $R_{r}=r^{-1}$, which is the case treated by Wimp. Theorems 4.3 and 4.5 , only with slight changes in notation and proofs, apply to Wimp's approximations $\bar{B}_{k}$ too.

5. Examples. In this section we shall show, through three typical examples, that the assumptions made in the previous sections are realistic and we shall especially be concerned with the application of Process II to these examples, keeping in mind the results of Section 4.

Example 1. $A_{r}=\Sigma_{m=1}^{r} z^{m-1} / m, r=1,2, \ldots$. This sequence satisfies the conditions of Theorem 2.2 with $\alpha=-1$ in (2.7); therefore Theorem 2.2 applies to it. Now $\lim _{r \rightarrow \infty} A_{r}=-(1 / z) \log (1-z)=F(z)$, provided $|z| \leqslant 1, z \neq 1 . \quad z=1$ is a branch point of $F(z)$ and we put the branch cut along the real interval $[1, \infty)$. This being the case, Theorem 3.1 applies and $T_{k, n}-F(z)=O\left(n^{-2 k-1}\right) z^{n}$ as $n \rightarrow \infty$.

Taking $z \notin[1, \infty)$ and integrating both sides of the equality

$$
\frac{1}{1-s}=\sum_{m=0}^{r-1} s^{m}+\frac{s^{r}}{1-s}
$$

from $s=0$ to $s=z$ along a straight line in the $s$-plane, and dividing by $z$, we obtain

$$
F(z)=A_{r}+\frac{1}{z} \int_{0}^{z} \frac{s^{r}}{1-s} d s .
$$

Letting $s=z e^{-\xi}$ in the integral on the right-hand side of (5.2), the contour in the $s$ plane is mapped to the positive real line in the $\xi$-plane, and (5.2) becomes

$$
F(z)=A_{r}+z^{r} \int_{0}^{\infty} e^{-r t}\left(e^{t}-z\right)^{-1} d t .
$$

Defining $R_{r}=z^{r-1} / r$, the $r$ th term of the infinite series $\Sigma_{m=1}^{\infty} z^{m-1} / m$, as in the $t$ transformation of Levin, we can express (5.3) in the form (1.5) with $f(x)=x \bar{f}(x)$, where $\bar{f}(x)=L[\phi(t) ; x]$ and $\phi(t)=z\left(z-e^{t}\right)^{-1}$. Since $\phi(t)$ is analytic at $t=0$ and for any $t>0$, provided $z \notin[1, \infty)$, applying (4.2) we therefore obtain 


$$
f(x) \sim \frac{z}{z-1}+\frac{z}{(z-.1)^{2}} \frac{1}{x}+\cdots,
$$

with $\beta_{0}=z /(z-1)$ as predicted by Theorem 2.1 , and this expansion is valid both for $|z| \leqslant 1, z \neq 1$, and for $|z|>1, z \notin[1, \infty)$, see the remark preceding the proof of Theorem 2.2.

Since $\bar{f}(p)$ is a Laplace transform, it is analytic for $\operatorname{Re} p>-1$, therefore so is $f(p)$. However, since $\phi(\xi)$ is not an entire function, (5.4) diverges for all $x$, hence $f(x)$ is not analytic at infinity. On the other hand, it is easy to show that $f(x)$ is infinitely differentiable at $x=\infty$. This is an important property that $f(x)$ was required to have in Process II in (I).

Now the function $\phi(\xi)$ is meromorphic and its only poles are $\xi=\log z+i 2 \pi l$, $l=0, \pm 1, \pm 2, \ldots$, i.e., all the singularities are on the straight line $\operatorname{Re} \xi=\log |z|$. Furthermore, $\phi(\xi)$ is uniformly bounded as $\operatorname{Re} \xi \rightarrow \infty$, in fact $|\phi(\xi)| \leqslant|z|\left(e^{t}-|z|\right)^{-1}$ $=O\left(e^{-t}\right)$ as $\operatorname{Re} \xi=t \rightarrow \infty$. Hence the strip $S(u)$ in Lemma 4.1 exists and $u$ in determined as follows: For $|z|<1, u=|\log | z|+i \arg z|-\delta$; for $|z| \geqslant 1, \arg z \neq$ $0, u=|\arg z|-\delta$ for $\delta>0$ and as small as we wish. Therefore, Theorem 4.2 applies and consequently (4.16) holds.

For example, for $z=-1, T_{k, n}-F(-1)=O\left[((\pi-\delta) e)^{-k}\right]$ at least, as $k \rightarrow \infty$. For this case the sequence $A_{r}, r=1,2, \ldots$, is a very slowly converging oscillatory sequence. For $z=-2, T_{k, n}-F(-2)=O\left[((\pi-\delta) e / \sqrt{2})^{-k}\right]$ at least, as $k \rightarrow \infty$, and for this case the sequence $A_{r}, r=1,2, \ldots$, is a strongly diverging oscillatory sequence.

Example 2. $A_{r}=\Sigma_{m=1}^{r} z^{m-1} / m^{2}, r=1,2, \ldots$. This sequence satisfies the conditions of Theorem 2.2 with $\alpha=-2$ in (2.7). Now the $A_{r}$ of this example are the partial sums of the Maclaurin series of the function

$$
F(z)=\frac{1}{z} \int_{0}^{z} \frac{\log (z / s)}{1-s} d s,
$$

where the integral is taken along the straight line in the $s$-plane, joining $s=0$ to $s=z$. Then $F(z)$ has a branch point at $z=1$ and a branch cut along the real interval $[1, \infty)$. By using the expansion in $(5.1)$, we can express $F(z)$ as follows:

$$
F(z)=A_{r}+\frac{1}{z} \int_{0}^{z} \frac{s^{r} \log (z / s)}{1-s} d s
$$

Making the change of variable $s=z e^{-\xi}$ in the integral on the right-hand side of (5.5), exactly as in the previous example, we obtain

$$
F(z)=A_{r}+z^{r} \int_{0}^{\infty} e^{-r t} \frac{t}{e^{t}-z} d t,
$$

where $t=\operatorname{Re} \xi$. Defining $R_{r}=z^{r-1} / r^{2}$ for $z \neq 1$, again as in the $t$-transformation of Levin, we obtain $f(x)=z x^{2} \int_{0}^{\infty} e^{-x t} t /\left(z-e^{t}\right) d t$, which, on using Watson's lemma for $x \rightarrow \infty$, becomes

$$
f(x) \sim \frac{z}{z-1}+\frac{2 z}{(z-1)^{2}} \frac{1}{x}+\cdots, \quad z \notin[1, \infty),
$$


as in the previous example. Hence, also for this example, we see that (1.5) and (1.6) are valid beyond the circle of convergence of $\Sigma_{m=1}^{\infty} z^{m-1} / m^{2}$. Using the fact that

$$
L\left[g^{\prime}(t) ; p\right]=p L[g(t) ; p]-g(0),
$$

we can express $f(x)$ in the form $f(x)=x \bar{f}(x)$, where $\bar{f}(x)=L[\phi(t) ; x]$ and $\phi(t)=$ $z\left[1 /\left(z-e^{t}\right)+t e^{t} /\left(z-e^{t}\right)^{2}\right]$. Now this $\phi(\xi)$ has the same properties as that $\phi(\xi)$ of the previous example. Therefore, the conclusion of the previous example concerning Process II is valid also for the present example.

We now want to investigate Process II for $z=1$, for which $\Sigma_{m=1}^{\infty} 1 / m^{2}$ is a monotonic series. For this case

$$
F(1)=A_{r}+\int_{0}^{\infty} e^{-r t} \frac{t}{\left(e^{t}-1\right)} d t .
$$

Choosing $R_{r}=1 / r$ as in the $u$-transformation of Levin, we have $f(x)=x \bar{f}(x)$, where $\bar{f}(x)=L[\phi(t) ; x]$, with $\phi(t)=t /\left(1-e^{t}\right)$. Again using Watson's lemma, we obtain

$$
f(x) \sim-\sum_{i=0}^{\infty} B_{i} / x^{i} \text { as } x \rightarrow \infty,
$$

where $B_{i}$ are the Bernoulli numbers. Again $\beta_{0}=-B_{0}=-1$, as predicted by Theorem 2.1. Now $\phi(\xi)$ satisfies all the conditions of Lemma 4.1 with $u=2 \pi-\delta$ and therefore the result of Theorem 4.3 holds and $T_{k, n}-F(1)=O\left((2 \pi-\delta)^{-k}\right)$ as $k \rightarrow \infty$.

Example 3. $A_{r}=\Sigma_{m=1}^{r} 1 / m-\log r, r=1,2, \ldots$ It is known that $\lim _{r \rightarrow \infty} A_{r}=C$, Euler's constant. Denoting $a_{1}=1, a_{r}=A_{r}-A_{r-1}=1 / r+$ $\log (1-1 / r), r=2,3, \ldots$, we can see that $a_{r}$ is as in Theorem 2.2, with $\alpha=-2$ and $z=1$. Therefore, (1.5) and (1.6) hold with $R_{r}=1 / r$, in accordance with (2.15).

Now let us show that, also for this case $f(x)=x \bar{f}(x)$, where $\bar{f}(x)=L[\phi(t) ; x]$ with $\phi(t)=t^{-1}-\left(e^{t}-1\right)^{-1}$.

Using the fact that $\psi(r+1)=-C+\Sigma_{m=1}^{r} 1 / m$ and Gauss' formula for the Psi function, see Olver (1974, pp. 39-40), we have

$$
\sum_{m=1}^{r} 1 / m=C+\int_{0}^{\infty}\left(\frac{e^{-t}}{t}-\frac{e^{-r t}}{e^{t}-1}\right) d t
$$

Now, for the integral on the right-hand side of (5.11), we can write

$$
\psi(r+1)=\int_{0}^{\infty}\left(\frac{e^{-t}}{t}-\frac{e^{-r t}}{e^{t}-1}\right) d t=\lim _{\epsilon \rightarrow 0+} \int_{\epsilon}^{\infty}\left(\frac{e^{-t}}{t}-\frac{e^{-r t}}{e^{t}-1}\right) d t .
$$

Making the change of variable $t=r t^{\prime}$ in the integral $\int_{\epsilon}^{\infty}\left(e^{-t} / t\right) d t$, we can express (5.12) as

$$
\psi(r+1)=\lim _{\epsilon \rightarrow 0+}\left[\int_{\epsilon}^{\infty} e^{-r t} \phi(t) d t+\int_{\epsilon / r}^{\epsilon} \frac{e^{-r t}}{t} d t\right]
$$

The second integral in (5.13) can easily be shown to be equal to $\log r+O(\epsilon)$ as $\epsilon \rightarrow 0+$. Letting now $\epsilon \rightarrow 0+$, the desired result follows. 
Now $\phi(\xi)$ is a meromorphic function with poles at $\xi=i 2 \pi l, l= \pm 1, \pm 2, \ldots$, and is uniformly bounded as $\operatorname{Re} \xi \rightarrow \infty$, actually $\phi(\xi)=O\left(\xi^{-1}\right)$ as $\operatorname{Re} \xi \rightarrow \infty$. Therefore, all the conditions of Lemma 4.1 are satisfied with $u=2 \pi-\delta$, for $\delta>0$ but as small as we wish. Hence (4.15) holds. Consequently, Theorem 4.3 holds and $T_{k, n}-C=O\left((2 \pi-\delta)^{-k}\right)$ at least, as $k \rightarrow \infty$.

Finally, we note that the $T$-transformation has been applied with great success to the sequences

$$
A_{r}=\sum_{m=1}^{r} \mu_{m} / z^{m}, \quad r=1,2, \ldots, \text { where } \mu_{m}=\int_{0}^{1} w(x) x^{m-1} d x,
$$

$m=1,2, \ldots$, and $w(x)=(1-x)^{\alpha} x^{\beta}(-\log x)^{\nu}, \alpha+\nu>-1, \beta>-1$. Actually, $A_{r}$ are the partial sums of the Laurent expansion at $z=\infty$ of the functions $F(z)=$ $\int_{0}^{1} w(x) /(z-x) d x$.

For these sequences it can be shown that

$$
\mu_{r} \sim \frac{1}{r^{\alpha+\nu+1}} \sum_{i=0}^{\infty} \frac{l_{i}}{r^{i}} \text { as } r \rightarrow \infty,
$$

which is of the form dealt with in Theorem 2.2. Furthermore, it can be shown that (2.16) is satisfied with $R_{r}=1 /\left(r^{\alpha+\nu+1} z^{r}\right)$ and for all $z \notin[0,1]$. The rational approximations, obtained by applying the $T$-transformation to these sequences, have been used to derive very accurate numerical quadrature formulas without preassigned abscissas for integrals with algebraic and logarithmic endpoint singularities of the form $\int_{0}^{1}(1-x)^{\alpha} x^{\beta}(-\log x)^{\nu} g(x) d x$. These formulas have the property that for some families of weight functions they have the same set of abscissas and they also have positive weights; for details see Sidi (1980).

The power series dealt with in this work, in particular in this section, fall in the category of (1) linearly convergent alternating series when $z \in(-1,0)$ (or $z \in[-1,0)$ if for $z=-1$ they converge), (2) linearly convergent monotonic series when $z \in$ $(0,1)$, and (3) logarithmically convergent (monotonic) series when $z=1$, if they converge. For such series (and others) different linear and nonlinear acceleration methods have been compared numerically by Smith and Ford (1979). Their conclusions for the series of this work, with respect to four nonlinear methods, namely Levin's $u$ transformation (i.e., the $T$-transformation with $R_{r}=r \Delta A_{r-1}$ ), the transformations of Shanks (1955) or their implementation, the $\epsilon$-algorithm of Wynn (1956), the $\rho$-algorithm of Wynn (1956a), and the $\theta$-algorithm of Brezinski (1971), are as follows: For linearly convergent alternating series the $u$-transformation is the best, followed by the $\theta$-algorithm and the $\epsilon$-algorithm. The $\rho$-algorithm fails to work. For linearly convergent monotonic series the $u$-transformation is again the best, followed by the $\epsilon$ algorithm and the $\theta$-algorithm. The $\rho$-algorithm again fails. For logarithmically convergent series the $\rho$-algorithm is usually the best, the $u$-transformation is slightly inferior and the $\theta$-algorithm is third best in efficiency. The $\epsilon$-algorithm fails to work for such series. As for the performance of linear methods, it turns out that they are usually less efficient than the nonlinear methods, which are applicable, and they have limited scope. 
Department of Computer Science

Technion-Israel Institute of Technology

Haifa, Israel

C. BREZINSKI (1971), "Accélération de suites à convergence logarithmique," C. $R$. Acad. Sci. Paris Sér. A-B, v. 273, pp. A727-A730.

E. ISAACSON \& H. B. KELLER (1966), Analysis of Numerical Methods, Wiley, New York, London.

D. LEVIN (1973), "Development of non-linear transformations for improving convergence of sequences," Internat. J. Comput. Math., v. B3, pp. 371-388.

D. LEVIN \& A. SIDI (1975), "Two new classes of non-linear transformations for accelerating the convergence of infinite integrals and series," Appl. Math. Comput. (To appear.)

F. W. J. OLVER (1974), Asymptotics and Special Functions, Academic Press, New York and London.

D. SHANKS (1955), "Non-linear transformations of divergent and slowly convergent sequences," J. Math. Phys., v. 34, pp. 1-42.

A. SIDI (1979), "Convergence properties of some nonlinear sequence transformations," Math. Comp., v. 33, pp. 315-326.

A. SIDI (1980), "Numerical quadrature and nonlinear sequence transformations; Unified rules for efficient computation of integrals with algebraic and logarithmic endpoint singularities," Math. Comp., v. 35, pp. 851-874.

D. A. SMITH \& W. F. FORD (1979), "Acceleration of linear and logarithmic convergence," SIAM J. Numer. Anal., v. 16, pp. 223-240.

I. H. SNEDDON (1972), The Use of Integral Transforms, McGraw-Hill, New York.

J. WIMP (1977), "New methods for accelerating the convergence of sequences arising in Laplace transform theory," SIAM J. Numer. Anal., v. 14, pp. 194-204.

P. WYNN (1956), "On a device for computing the $e_{m}\left(S_{n}\right)$ transformation," MTAC, v. 10, pp. 91.96 .

P. WYNN (1956a), "On a procrustean technique for the numerical transformation of slowly convergent sequences and series," Proc. Cambridge Philos. Soc., v. 52, pp. 663-671. 